\title{
COVID-19 and the Management of Chronic Mental Illnesses
}

\author{
Afzal Javed - Maryam Afzal
}

Received: 16 July 2020/ Accepted: 17 July 2020/Published online: 27 July 2020

(C) Springer Nature India Private Limited 2020

The current coronavirus pandemic has changed the world as we knew it. Unlike many pandemics, Covid19 has affected not only the health sector but has also caused several implications for the social and financial sectors as well. Looking at the health implications, there is no age that is immune to this infection but there have been more concerns for vulnerable populations.

Mental health is also hit by this pandemic and in many ways it is at the frontline that addressing of emotional and social aspects of this scourge requires urgent attention [1]. The pandemic has resulted in a surge of patients with first episode, acute exacerbation and relapses of pre-existing disorders along with relapses of psychiatric disorders like depression, anxiety, panic disorders and even acute psychosis. Fear of emerging and re-emerging infections is affecting the general population, but mentally ill patients are becoming more vulnerable especially due to their difficulties in coping with stressors and uncertainties.

A. Javed ( $\square)$

Pakistan Psychiatric Research Centre, Fountain House,

37-Lower Mall, Lahore, Pakistan

e-mail: afzalj@gmail.com

M. Afzal

Aneurin Bevan University Health Board, 6 Goldtops,

Newport NP20 4PG, UK
Whereas, the COVID-19 pandemic has brought new challenges for the management of psychiatric patients and the administration of psychiatric treatments, the interface of psychiatry and COVID-19 is relevant for both COVID positive and negative cases [2]. There are many areas in psychiatric treatment especially for those who are suffering from chronic to enduring conditions, where caution has to exerted during the pandemic. While vigilance must be exerted by medical specialists and psychiatrists when handling acute problems during the current situation, there is a strong need to look at the chronic mentally ill who are living in the community and are not attending any long-term or ongoing psychosocial rehabilitation plans. Many of such patients (unlike other patients) will need extra help from a psychosocial point of view to combat the COVID-related plus mental illnessrelated stigma. They may need special assistance for supervised medication, financial security, safe housing, and sound social support. With the closure of many day centres and drop-in centres during the COVID-19 pandemic, these patients are certainly facing a lot of sufferings to manage their problems with limited and inadequate coping skills.

Jonathan et al. [3] rightly reported that the COVID19 pandemic presents a triple global public mental health challenge. This involves preventing associated increase in mental disorders and a reduction in mental wellbeing across populations, protecting people with a 
mental disorder from COVID-19, and providing appropriate public mental health interventions to health professionals and carers.

Although the psychiatric community responded to the expected psychosocial problems produced by the coronavirus, patients with mental illness, unfortunately are generally neglected in many parts of the world. It is hoped that future strategies and subsequent steps dealing with pandemics like COVID-19 will also look at the services for patients with chronic mental illness who require continuous support for psychosocial rehabilitation [4].

\section{References}

1. Tedros AG. Addressing mental health needs: an integral part of COVID-19 response. World Psychiat. 2020;19(2):129-30.

2. Javed A, Mohandas E, De-Sousa A. The interface of psychiatry and COVID-19: challenges for management of psychiatric patients. Pak J Med Sci. 2020;36(5):1133-6. https:// doi.org/10.12669/pjms.36.5.3073.

3. Campion J, Javed A, Sartorius N, Marmot M. Addressing the public mental health challenge of COVID-19. Lancet Psychiatry. 2020. https://doi.org/10.1016/S22150366(20)30240-6.

4. Chaturvedi SK. Covid-19, coronavirus and mental health rehabilitation at times of crisis. J Psychosoc Rehabil Mental Health. 2020;7:1-2. https://doi.org/10.1007/s40737-02000162-z.

Publisher's Note Springer Nature remains neutral with regard to jurisdictional claims in published maps and institutional affiliations. 